\title{
Nitric Oxide Influences Injury-Induced Microglial Migration and Accumulation in the Leech CNS
}

\author{
Aileen Chen, ${ }^{1}$ Shanta M. Kumar, ${ }^{2}$ Christie L. Sahley, ${ }^{2}$ and Kenneth J. Muller ${ }^{1}$ \\ ${ }^{1}$ Department of Physiology and Biophysics, University of Miami School of Medicine, Miami, Florida 33136, and \\ 2Department of Biological Sciences, Purdue University, West Lafayette, Indiana, 47907
}

Damage to the leech or mammalian CNS increases nitric oxide (NO) production and causes accumulation of phagocytic microglial cells at the injury site. The aim of this study was to determine whether NO plays a role in microglial migration and accumulation at lesions in which NO is generated by a rapidly appearing endothelial nitric oxide synthase (eNOS) in leeches. Immunohistochemistry and cytochemistry demonstrated active eNOS before and throughout the period of microglial accumulation at the lesion. Decreasing NO synthesis by application of the NOS inhibitor $N^{w}$-nitro-L-arginine methyl ester (1 mM) significantly reduced microglial accumulation, whereas its inactive enantiomer $N^{\mathrm{w}}$-nitro-D-arginine methyl ester $(1 \mathrm{~mm})$ resulted in microglial accumulation similar to that in crushed controls. Increasing $\mathrm{NO}$ with the donor spermine NONOate (SPNO) (1 mM)

One of the first responses to CNS injury is by microglial cells, the resident macrophages of the brain, which retract their branches, become amoeboid, and migrate to the site of damage. The migration leading to accumulation is thought to be triggered by substances including cytokines released at the damage site, but few studies have examined migration of microglia within the living nervous system. The role of microglia in recovery is controversial, because although they may produce harmf ul cytotoxins that induce neuronal death (Thanos et al., 1993; Barron, 1995; Angelov et al., 1998), they may also secrete trophic factors that enhance neuronal survival (Barron, 1995; Elkabes et al., 1996) and components of extracellular matrix, such as laminin, that promote growth (von Bernhardi and Muller, 1995). It has been shown in the leech that immediately after injury, even before microglia accumulate, activity of the constitutive endothelial nitric oxide synthase (eNOS) increases and that some injured microglia, as well as the injured region of the connective glia, express eNOS (Shafer et al., 1998). Significantly, the eNOS immunoreactivity increases in parallel with NOS activity and reflects activation of eNOS. An upregulation of NOS has also been observed in certain diseased states of mammalian CNS and after peripheral nerve injury, although in general this increase is in the inducible nitric oxide synthase isoform and is delayed by hours to days (Blottner et al., 1995).

\footnotetext{
Received Aug. 27, 1999; revised Nov. 8, 1999; accepted Nov. 11, 1999.

This work was supported by National Institutes of Health Grants NS34927 and NS37025 to K.J.M. and C.L.S. We thank Orie Shafer for conducting preliminary experiments, Shirly Mildner for counting microglia, and Susanna Blackshaw for instruction in making collagen gels.

Drs. Chen and Kumar contributed equally to this work.

Correspondence should be addressed to Dr. Kenneth J. Muller, Department of Physiology and Biophysics (R-430), University of Miami School of Medicine, Miami, FL 33136. E-mail: kmuller@newssun.med.miami.edu.

Copyright (C) 2000 Society for Neuroscience $0270-6474 / 00 / 201036-08 \$ 15.00 / 0$
}

also inhibited accumulation, but not in the presence of the NO scavenger 2-(4-carboxyphenyl)-4,4,5,5-teramethylimidazolineoxyl-3-oxide $(50 \mu \mathrm{M})$. The effect of SPNO was reversed by washout. SPNO application reduced average microglial migratory speeds and even reversibly arrested cell movement, as measured in living nerve cords. These results suggest that NO produced at a lesion may be a stop signal for microglia to accumulate there and that it can act on microglia early in their migration. Thus, NO may assume a larger role in nerve repair and recovery from injury by modulating accumulation of microglia, which appear to be important for axonal regeneration.

Key words: nitric oxide; cell migration; endothelial nitric oxide synthase; Hirudo medicinalis; nerve repair; nerve regeneration; microglia
Nitric oxide (NO) influences motile cells in both vertebrates and invertebrates. It inhibits migration of rat vascular smooth muscle cells (Sarkar et al., 1996) and active amoeboid invertebrate immunocytes and microglia, which become rounded (Magazine et al., 1996). The NO-induced changes in morphology and motility, thought to be mediated principally by activation of guanylate cyclase, might be modulated through changes in cell adhesion resulting from disruption of the cytoskeleton (Frenkel et al., 1996; Yao et al., 1998).

The injured leech CNS, in which synapse regeneration is successful (Camhi and Macagno, 1991; Nicholls, 1987), was examined to determine whether NO could influence the migration and accumulation of microglial cells (Stewart, 1994). Leech microglia are believed to produce laminin, which promotes axon outgrowth (von Bernhardi and Muller, 1995). Microglia in the segmented nerve cord of the leech can be identified unambiguously without special markers, because the only other nucleated cells among the thousands of axons that extend between segmental ganglia are the two large glial cells, up to $5 \mathrm{~mm}$ in length, that ensheathe the axons and are located midway between ganglia. Thus, microglia can be tracked in the living cord with the aid of fluorescent nuclear dyes (McGlade-McCulloh et al., 1989). The relationship between NO and microglial migration and accumulation after CNS injury was examined because NOS (1) is rapidly activated at the lesion, (2) influences migrating cells, and (3) is generated by microglia themselves (Shafer et al., 1998).

Portions of this paper have been published previously in abstract form (Chen et al., 1998).

\section{MATERIALS AND METHODS}

Preparation and operations. Adult 4-6 gm adult leeches (Hirudo medicinalis) were obtained from a commercial supplier (Leeches USA, Westbury, NY) and maintained in artificial pond water (Forty Fathoms, 0.5 $\mathrm{gm} / 1 \mathrm{H}_{2} \mathrm{O}$; Marine Enterprises, Towson, MD) at $22^{\circ} \mathrm{C}$. For experiments, 
individual segmental ganglia with connectives or, for time-lapse video microscopy, isolated connectives were dissected in physiological saline (Nicholls and Baylor, 1968) and pinned in a dish coated with silicone rubber (Sylgard 184; Dow Corning, Midland, MI). When periods of incubation were required, as after crushing, preparations were placed in Leibowitz-15 culture medium supplemented with $2 \%$ fetal calf serum, $0.6 \%$ glucose, and gentamicin $(10 \mathrm{mg} / \mathrm{ml})$ (Ready and Nicholls, 1979), referred to here as L-15. Pharmacological reagents were dissolved in L-15 or physiological saline, as indicated.

Staining of eNOS and microglial cells. To examine the distribution of eNOS immunoreactivity and microglia at sites of injury, crushed cords were immunostained with monoclonal antibodies to human eNOS (Transduction Laboratories, Lexington, KY), and microglial nuclei then stained with Hoechst 33342 dye. In brief, connectives were dissected, crushed with a pair of fine forceps (Dumont \#5), and left at room temperature for $5 \mathrm{~min}, 3 \mathrm{hr}, 6 \mathrm{hr}$, or $24 \mathrm{hr}$ in L-15. The tissue was then fixed in $4 \%$ paraformaldehyde for $20 \mathrm{~min}$, incubated $30 \mathrm{~min}$ in a blocking solution $[10 \%(\mathrm{v} / \mathrm{v})$ fetal calf serum and $2 \%(\mathrm{v} / \mathrm{v})$ Triton X-100 in PBS, $\mathrm{pH}$ 7.5], and then kept overnight at $4^{\circ} \mathrm{C}$ in anti-eNOS monoclonal antibody diluted 1:150 in blocking solution. For the secondary antibody, a 1:200 dilution of Texas Red-conjugated rabbit anti-mouse IgG (heavy and light chain; Molecular Probes, Eugene, OR) was used. The tissue was mounted in glycerol with Hoechst 33342 dye $(10 \mu \mathrm{g} / \mathrm{ml})$ and viewed with a Zeiss (Oberkochen, Germany) epifluorescence microscope and appropriate filters.

Determining the effect of NOS inhibition on accumulation. Microglial accumulation was investigated in the presence of $N^{\mathrm{w}}$-nitro-L-arginine methyl ester (L-NAME), a general NOS inhibitor. In brief, nerve cords were crushed in a standard manner, incubated in 0.01-1 mM L-NAME or $N^{\mathrm{w}}$-nitro-D-arginine methyl ester (D-NAME), the inactive enantiomer of L-NAME, in leech saline with $10 \mathrm{~mm}$ glucose for $6 \mathrm{hr}$, fixed $30 \mathrm{~min}$ in $4 \%$

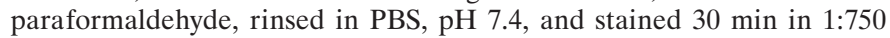
Yo-Pro-1 (Molecular Probes). The Yo-Pro-1 dye was used because it is an argon-ion laser excitable nucleic acid stain that allowed us to view a series of optical sections of washed tissue with a Bio-Rad (Hercules, CA) laser scanning confocal microscope equipped with fluorescein and rhodamine optics and a Nikon (Tokyo, Japan) $20 \times$ objective. The accumulation of microglia was analyzed at the crush over an area of $\sim 0.08 \mathrm{~mm}^{2}$, by capturing a $\mathrm{Z}$ series consisting of $252 \mu \mathrm{m}$ optical sections through the connectives. For each sample, microglia at the site of injury were counted in every fifth section, and the average number of microglia in the five sections was determined. Statistical significance was determined by ANOVA, followed by post hoc analysis of the significant main effects.

Determining the effect of exogenous NO on accumulation. The NO donor spermine NONOate (SPNO) (Calbiochem, La Jolla, CA) was used to determine the effect of exogenous NO on microglial accumulation. Stock solutions of $100 \mathrm{~mm}$ SPNO $(100 \times)$ were prepared in sodium phosphate buffer, $\mathrm{pH} 8.5$, and stored at $-70^{\circ} \mathrm{C}$ in $10 \mu \mathrm{l}$ aliquots. Crushed cords were incubated in $1 \mathrm{~mm}$ SPNO in L-15 culture medium for $3 \mathrm{hr}$, and microglial nuclei were labeled with Feulgen's nucleic acid-specific stain, using the method of Morgese et al. (1983). The Feulgen stain was used to measure accumulation at the highest concentration of cells and was more permanent than fluorescent stains. Control cords were incubated in L-15. In some cases, the NO scavenger 2-(4-carboxyphenyl)4,4,5,5-teramethylimidazoline-oxyl-3-oxide (carboxy-PTIO or cPTIO) $(50 \mu \mathrm{M})$ was included with $1 \mathrm{~mm}$ SPNO to ensure that the effects of SPNO were because of NO and not some other breakdown product of SPNO.

Once an effect of SPNO on microglial accumulation had been determined, the reversibility of the effect of SPNO was examined. For these experiments, individual ganglia with their attached connectives were dissected intact and placed in L-15 alone (controls) or with 1 mM SPNO. Connectives were crushed by fine forceps anterior and posterior to ganglia and placed in three separate groups. First, as controls, the crushed preparations were incubated in L-15 for $6 \mathrm{hr}$. Second, crushed preparations were incubated in $1 \mathrm{~mm}$ SPNO for $6 \mathrm{hr}$. Third, to determine whether the effect of SPNO was reversible, a crushed group was incubated $3 \mathrm{hr}$ in SPNO and then washed $3 \mathrm{hr}$ in L-15.

To quantify peak accumulation for each group, the following sampling technique was used with Feulgen-stained tissue, referred to here as a "line count." A hairline in a microscope ocular was positioned perpendicular to the longitudinal axis of the crush, and the number of nuclei within the sheath touching or intersecting the line in a through-focus series at the crush was then counted in a double-blind manner. Counts were made for both connectives at the anterior and posterior margins of the crush in which accumulation was highest, three series each. The counts were averaged and then analyzed using an ANOVA for repeated measures. Post hoc comparisons of the significant effects were assessed with a Newman-Keuls post hoc test. This method measures accumulation at the highest concentration of cells.

Low-light video microscopy. To determine the effect of L-NAME, D-NAME, and SPNO on microglial movement in injured preparations, individual live Hoechst-stained cells were tracked using low-light video microscopy. Procedures were basically those of McGlade-McCulloh et al. (1989). In brief, 2-3 mm pieces of connective were dissected, stained 10-30 min with Hoechst 33342 dye [0.001\% (wt/vol) in leech saline], and pinned overnight at $16^{\circ} \mathrm{C}$ in L-15 with supplements. Although some microglia accumulate at the cut ends, by the time the cord is crushed the next day, the microglia are stationary and uniformly distributed, except for a local accumulation at the severed ends. Just before recording, the connectives were crushed and placed in a saline with $23 \mathrm{~mm} \mathrm{MgCl} \mathrm{M}_{2}$ replacing $\mathrm{NaCl}$ mole for mole to reduce muscle movement. To view moving cells, each preparation was illuminated with a Zeiss $12 \mathrm{~V}, 100 \mathrm{~W}$ tungsten-halogen lamp in a $350 \mu \mathrm{m}$ length of connectives $\sim 50 \mu \mathrm{m}$ from the crush. To minimize UV illumination of the tissue, a $30 \%$ neutral density filter and a computer-controlled shutter were placed in the path of the excitation beam. Images were captured and enhanced at $2.5 \mathrm{~min}$ intervals with a CCD camera and Argus-10 low-light enhancement image processor (Hamamatsu, Hamamatsu City, Japan) and Image 1/AT imaging software (Universal Imaging Corporation, Media, PA). For each cord, a baseline was recorded, followed by treatment with $1 \mathrm{~mm}$ L-NAME, 1 mM D-NAME, or $1 \mathrm{~mm}$ SPNO and washout of the drugs. For analysis, cells were selected that were visible through all three $30 \mathrm{~min}$ treatment periods. Average cell speed was then calculated by measuring the distance traveled by the same individual cells during each $30 \mathrm{~min}$ treatment period ( $n=9$ cells total through all treatments, and $n=6$ and 3 from two cords, respectively). The results were statistically analyzed as described for the microglia accumulation experiment.

Movement of microglia in three-dimensional collagen gels. Threedimensional (3-D) collagen gels (Guthrie and Lumsden, 1994) were adapted for leech at room temperature (Blackshaw et al., 1997) and examined with phase microscopy to observe directly the effects of NO donors on microglial movement. In brief, segments of nerve cord, including ganglia, were cultured in sterile collagen gels in supplemented L-15 culture medium up to $10 \mathrm{~d}$, during which time microglia migrated out of the cord into the transparent surrounding gel (Blackshaw et al., 1997). For experiments with the NO donor SPNO, the drug was added to a final concentration of $1 \mathrm{~mm}$ after an initial period of observation in L-15 culture medium and cleared after $30 \mathrm{~min}$. As a control, the NO scavenger-reduced hemoglobin $(\mathrm{Hb})$ was added to dishes either before or after SPNO exposure. Hb was reduced using methods of Rentería and Constantine-Paton (1995) with slight modification. In brief, bovine $\mathrm{Hb}$ (Sigma, St. Louis, MO) was diluted to $1 \mathrm{~mm}$ in distilled water and then chemically reduced by the addition of 10 -fold sodium hydrosulfite. The $\mathrm{Hb}$ solution was dialyzed overnight at $4^{\circ} \mathrm{C}$ in distilled water and then added to experimental dishes to a final concentration of $0.02 \mathrm{~mm}$.

\section{RESULTS}

\section{Nitric oxide synthase and microglial cell accumulation colocalize at sites of CNS injury}

To determine the spatial and temporal relationship of injuryinduced nitric oxide synthase and accumulating microglial cells, nerve cords were double-stained with a monoclonal antibody for human eNOS and with the fluorescent nuclear dye Hoechst 33342. In uncrushed cords, eNOS immunoreactivity is absent (Shafer et al., 1998), and microglial cells are evenly distributed throughout the connectives (Morgese et al., 1983). Cords fixed 5 min after a crush showed eNOS immunoreactivity at the lesion (Shafer et al., 1998) (Fig. 1A) but no accumulation of microglia within the crush (Fig. $1 B$ ). Three hours after crushing, however, eNOS immunoreactivity remained at the crush (Shafer et al., 1998) (Fig. 1C), and microglial cells had clustered there (Fig. 1D). At $24 \mathrm{hr}$, eNOS immunoreactivity extended slightly beyond the crush region and appeared more diffuse than at $3 \mathrm{hr}$ (Fig. 1E). Microglial cells also occupied a larger region expanding to areas outside the crush (Fig. $1 F$ ). Although both eNOS immunoreactivity and microglial cell accumulation were localized to the crush 

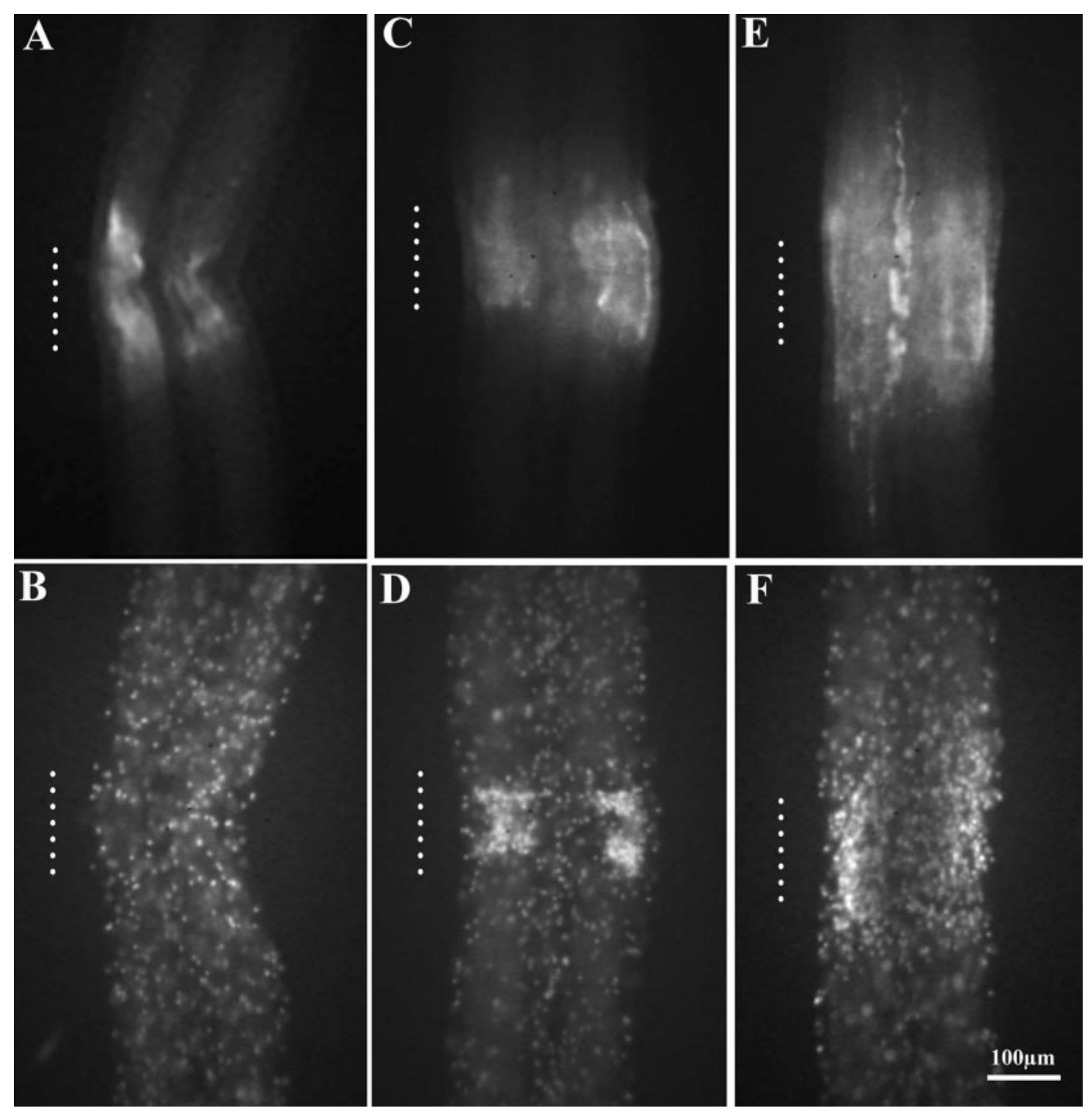

Figure 1. Appearance of eNOS immunoreactivity and microglial cell accumulation at sites of CNS damage. The connectives connecting ganglia are paired, with the bundled axons of each connective surrounded by a cellular sheath. Adult leech nerve cords were crushed and double-stained with a monoclonal antibody against human eNOS and with the fluorescent nuclear dye Hoechst 33342. The approximate longitudinal extent of the crushes is indicated by vertical dotted lines. Five minutes after crushing, eNOS immunoreactivity was present among nerve fibers in the connectives $(A)$, but microglial cells were still evenly distributed throughout the connectives $(B)$, resembling controls. Note that immunoreactivity was predominantly where crushed axons, glia, and microglia were located and not in the sheath. Three hours after injury, eNOS immunoreactivity persisted at the lesion $(C)$, and microglial cells had accumulated $(D)$. After $24 \mathrm{hr}$, eNOS immunoreactivity was more diff use and expanded outside the crush $(E)$. Microglial cells also occupied a larger area $(F)$.

site, their spatial distributions were not identical. The region of eNOS immunoreactivity was broader, extending beyond the high density of microglia. Thus, after injury, there was an immediate appearance of nitric oxide synthase immunoreactivity that persisted during later stages of microglial accumulation.

\section{NOS inhibitors block microglial cell accumulation}

To determine how NO production at the crush affects microglial cells, NO production was blocked with the NOS inhibitor L-NAME. Microglial cell accumulation in cords was examined 4-6 hr after injury. As controls, crushed cords were incubated in similar concentrations of D-NAME. Microglia accumulated in 1 mM D-NAME (Fig. $2 A$ ) but not in 1 mM L-NAME (Fig. $2 B$ ). Even at lower concentrations of L-NAME $(100-750 \mu \mathrm{M})$, accumulation was reduced compared with control cords incubated in leech saline containing $10 \mathrm{~mm}$ glucose alone or in D-NAME (data not shown).
To quantify the effect of NOS inhibition on microglial cell accumulation, microglial cells were counted in a series of planes through the lesion after incubating crushed, living cords $6 \mathrm{hr}$ in L-NAME, D-NAME, or saline vehicle alone. Crushed cords treated with $1 \mathrm{mM}$ L-NAME had counts similar to uncrushed cords, with significantly fewer cells accumulated at the lesion site than crushed controls. In the presence of $1 \mathrm{mM}$ L-NAME, the average \pm SEM number of cells accumulated at a crush site was $18.6 \pm 1.8$ cells, a level similar to noncrushed cords $(14.2 \pm 1.8)$, and significantly lower than control cords incubated in saline with glucose $(99.8 \pm 32.6)$ or $1 \mathrm{~mm}$ D-NAME $(105.6 \pm 25.3)$, the inactive enantiomer of L-NAME ( $n=4$ for each treatment) (Fig. 3 ). There was a significant difference between the crushed cords treated with $1 \mathrm{mM}$ L-NAME and control cords not exposed to drug treatment (ANOVA; Scheffe's test; $F_{(3,12)}=5.848 ; p=$ $0.0106 ; p<0.05)$. Because inhibition of NOS activity reduced the 


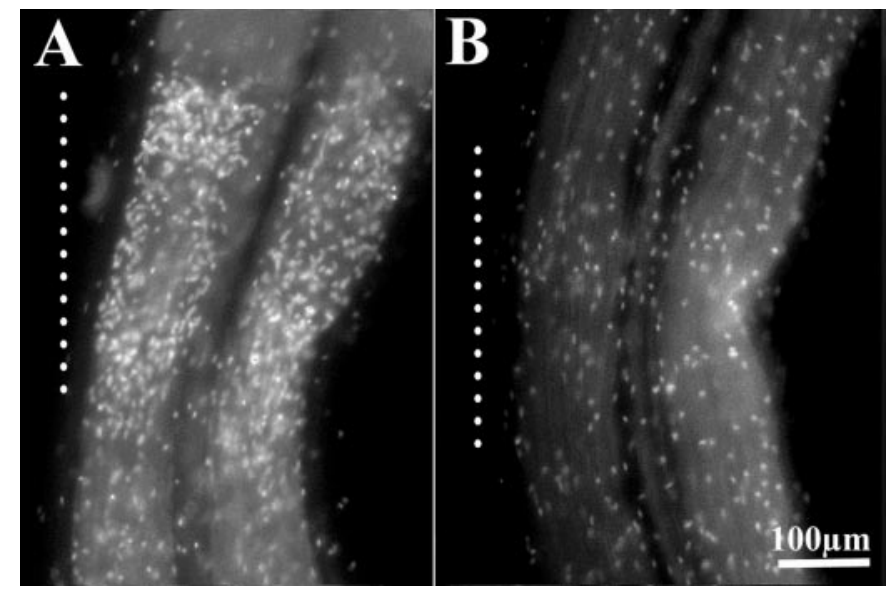

Figure 2. NOS inhibition blocked microglial accumulation. Adult leech nerve cord crushed $6 \mathrm{hr}$ before fixing and stained with the fluorescent nuclear stain Hoechst 33342 to show microglia. Vertical dotted lines indicate longitudinal extent of crush. D-NAME $(1 \mathrm{mM})$, the inactive enantiomer of L-NAME, did not block microglial accumulation at the crush $(A)$, whereas $1 \mathrm{mM}$ L-NAME blocked microglial accumulation $(B)$.

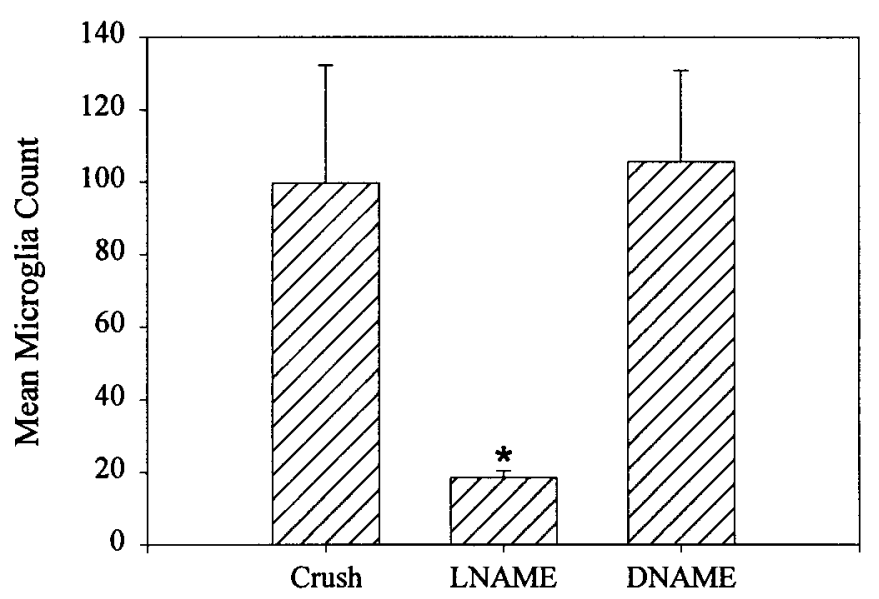

Treatment

Figure 3. L-NAME significantly reduced microglial accumulation. In 1 mM L-NAME, significantly fewer cells accumulated at the lesion site than in crushed controls (Crush) and were instead similar to uncrushed controls (data not shown). In contrast, in $1 \mathrm{mM}$ D-NAME, an inactive isoform of L-NAME, microglia accumulated at the crush site in numbers equivalent to crushed control cords. For each sample nerve cord, the average number of microglia at the crush site was determined (see Materials and Methods). There was a significant difference between microglial cell accumulation at the lesion site in injured tissue treated with1 mM L-NAME compared with tissue treated with $1 \mathrm{mM}$ D-NAME (ANOVA; Scheffe's test; $\left.F_{(3,12)}=5.848 ; p=0.0106 ; n=4\right)$.

microglial cell accumulation to levels comparable with those in uncrushed cords, it appears that the NO produced after injury influenced the aggregation of microglial cells at the site of injury.

\section{NO donors block microglial cell accumulation}

If microglia aggregate at a lesion as a result of locally increased $\mathrm{NO}$, then a general increase in NO might disrupt the injuryinduced NO gradient and interfere with accumulation. Crushed nerve cords were bathed in a $1 \mathrm{~mm}$ solution of SPNO, a NO donor with a relatively long half-life $\left(t_{1 / 2}=4 \mathrm{hr}\right.$ at $\left.25^{\circ} \mathrm{C}\right)$ for $3 \mathrm{hr}$, and control cords were bathed in L-15. Control cords incubated in L-15 for $3 \mathrm{hr}$ had large numbers of cells gathered at the lesion

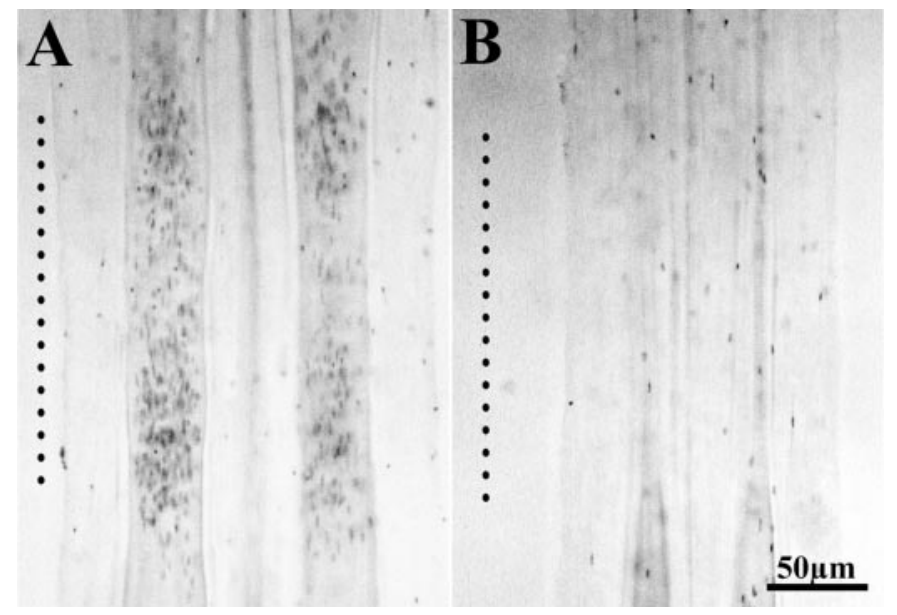

Figure 4. NO donor SPNO blocked microglial accumulation. A, Control. Without drug, there was a marked increase in microglia at the lesion (dotted line) inside the sheath by $3 \mathrm{hr}$. B. Exposure to $1 \mathrm{~mm}$ SPNO for 3 $\mathrm{hr}$ blocked the accumulation of microglia at the lesion. Cell nuclei were Feulgen-stained.

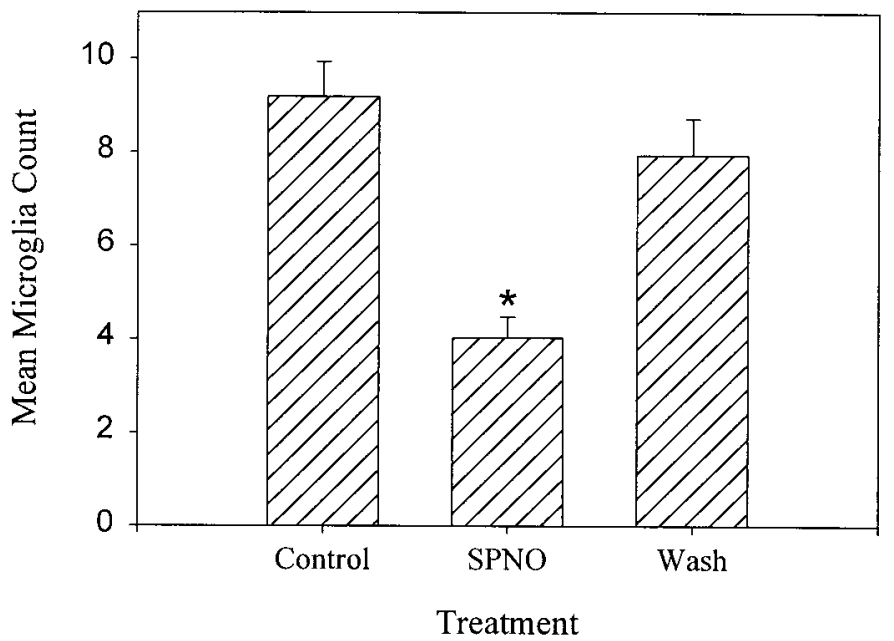

Figure 5. Quantitative comparison of the effect of SPNO on microglial accumulation. SPNO inhibited microglial cell accumulation, and this effect was reversible. Nerve cords were crushed and incubated in culture medium (Control) for $6 \mathrm{hr}, 1 \mathrm{~mm}$ SPNO for $6 \mathrm{hr}(S P N O)$, and $1 \mathrm{~mm}$ SPNO for $3 \mathrm{hr}$ followed by a $3 \mathrm{hr}$ wash in L-15 (Wash). Accumulation was measured by counting microglial cell nuclei that intersected a line at right angles to the long axis of the connectives (see Materials and Methods). The SPNO treatment significantly reduced microglial accumulation at the lesion site compared with controls. Washed cords (Wash) had cell counts similar to crush controls that sat for $6 \mathrm{hr}$ in L-15 (Control) but were significantly different from cords that were incubated in $1 \mathrm{~mm}$ SPNO for $6 \mathrm{hr}(S P N O)$ (ANOVA; Scheffe's test; $F_{(2,6)}=20.87 ; p<0.002$; NewmanKeuls post hoc; $p<0.05 ; n=5)$. Error bars are SEM. Groups significantly different from control are indicated by *.

(Fig. 4A), whereas cords incubated in the NO donor SPNO had significantly less accumulation there (Fig. 4B). As indicated in Figure 5, control cords incubated in L-15 for $3 \mathrm{hr}$ had large numbers of cells gathered at the lesion (line count, $9.2 \pm 0.7$ microglia; $n=5$ cords), whereas cords incubated in the NO donor SPNO had significantly less accumulation at the crush $(4.0 \pm 0.4$ microglia; $n=3$ cords). In cords that were treated with SPNO and then washed, microglia accumulated at the crush $(8.0 \pm 0.8$ microglia; $n=2$ cords). No significant differences were observed 
between the baseline and wash conditions (ANOVA; Scheffe's test; $F_{(2,6)}=20.87 ; p<0.002$; Newman-Keuls post hoc; $\left.p<0.05\right)$.

To determine that the effects of SPNO were caused by released NO and not SPNO degradation products, experiments were conducted with aged SPNO, which would have been expected to have a diminished effect. From a single animal, ganglia were exposed $3 \mathrm{hr}$ to $1 \mathrm{~mm}$ SPNO depleted by having been prepared $36 \mathrm{hr}$ earlier. With a line count, an average of $5.7 \pm 0.2$ microglia was measured ( $n=8$ crushes), which was more cells accumulated at the crush than in segments of cord bathed in fresh SPNO (3.6 \pm 0.2 microglia; $n=4$ crushes) but was fewer cells than in controls (8.3 $\pm 0.3 ; n=4$ crushes). Thus, partially expired SPNO was less potent than a fresh solution in blocking accumulation. Incubation of $1 \mathrm{~mm}$ SPNO with $0.5 \mathrm{~mm}$ cPTIO, a scavenger of NO in the solution, was sufficient to prevent blocking of microglial cell accumulation ( $8.0 \pm 0.1$ microglia; $n=12$ crushes $)$. Accumulation of microglia in cPTIO was not different from crushed control cords, showing that cPTIO blocks SPNO inhibition of accumulation and suggesting that the SPNO-induced inhibition of microglial cell accumulation occurred via a mechanism involving NO.

\section{SPNO but not L-NAME blocks microglial cell accumulation by stopping or slowing migration}

Video microscopy of living microglia in situ was used to determine why they did not accumulate when NO levels were manipulated. Among the possibilities were that the drug (1) stopped migration, (2) disoriented cells, changing their direction but not rate of movement, or (3) blocked the usual stop signals, causing cells to move through the lesion. With time-lapse video microscopy and low-light fluorescent illumination, live Hoechst-stained microglial nuclei were tracked in crushed connectives (McGladeMcCulloh et al., 1989) to test these possibilities. After a $30 \mathrm{~min}$ baseline to establish the directed movement of cells toward the lesion, migrating cells in the cord were exposed to $1 \mathrm{~mm}$ L-NAME, $1 \mathrm{~mm}$ D-NAME, or $1 \mathrm{~mm}$ SPNO and tracked for an additional $30 \mathrm{~min}$. The drug was then washed out, and preparations were observed for $30 \mathrm{~min}$ more to determine reversibility. The average distance traveled by each cell that stayed visible through the entire recording was determined for each treatment period. Comparisons with cells tracked during only one phase of the treatment indicated that the recorded cells were representative; movements are principally along one dimension, so travel was measured as distance relative to location at the beginning of observation or treatments. The fraction of microglia moving was within the range of $15-40 \%$ reported previously (McGladeMcCulloh et al., 1989), with no evidence of variation across the field of view, consistent with observations (K. J. Muller, unpublished observations) that microglia $>500 \mu \mathrm{m}$ from the lesion begin moving within 5 min of cord injury.

Overall, migrating cells stopped or slowed after SPNO exposure; however, a range of behaviors was observed. Figure 6, with each symbol representing a separate cell of six observed, shows the locations of cells at 10 min intervals relative to the start of the treatment period, with positive values representing displacement toward the lesion. Some cells moved toward the crush at variable speeds, even in baseline recordings (top panel). In SPNO (middle panel), the initial responses of cells ranged from immediate stopping (cells 2 and 5) to slowing little (e.g., cell 3). However, toward the end of the SPNO incubation period, all cells slowed if they had not stopped. After washout of SPNO, microglia overall increased their travel (bottom panel). One nucleus (5) initially moved away from the crush, a behavior reported previously for

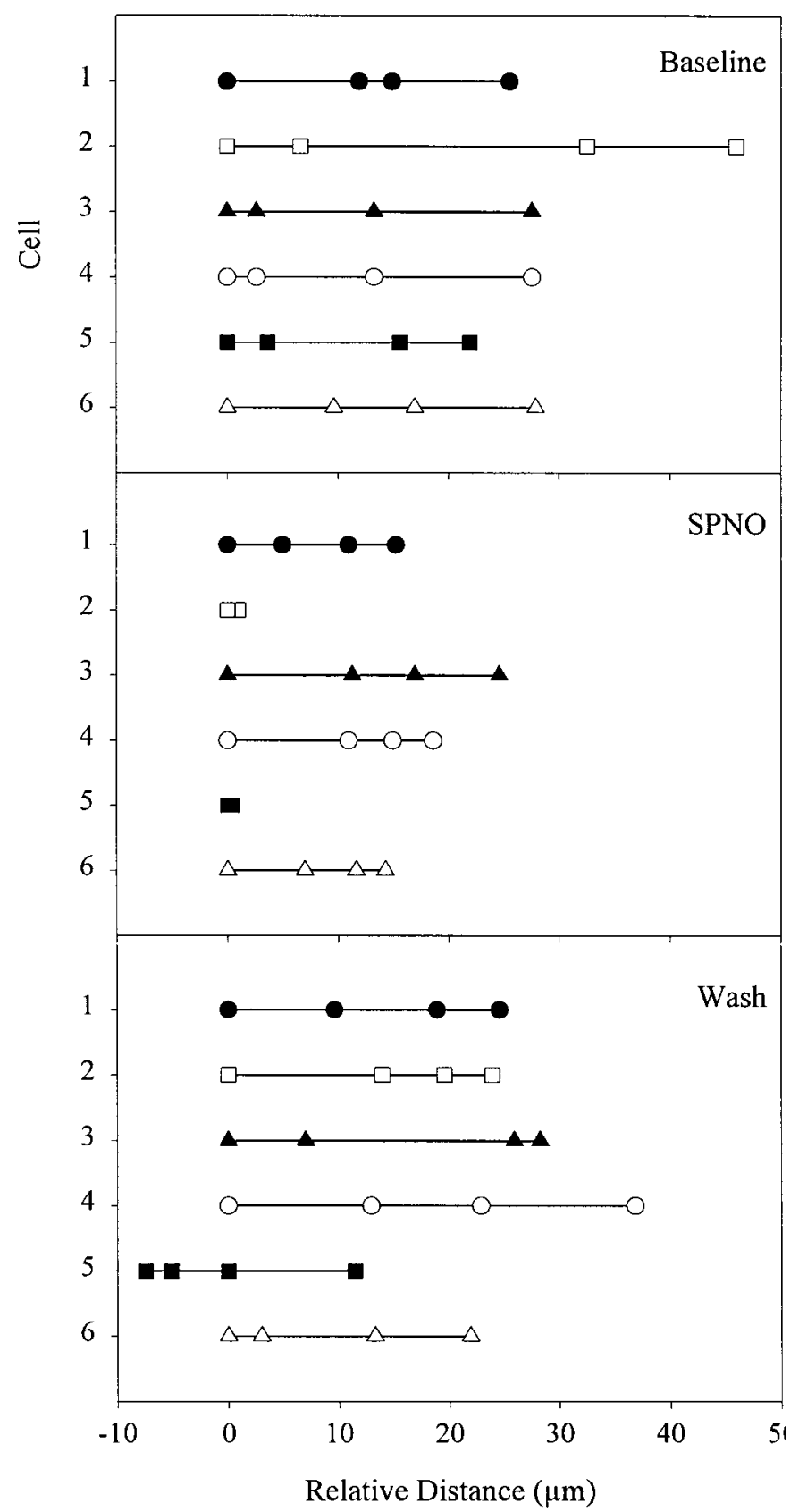

Figure 6. NO slows migrating microglial cells. The distances traveled by six different microglial cells (indicated by 6 symbols and numbers $1-6$ on the abscissa) were measured by low-light fluorescence video microscopy at $10 \mathrm{~min}$ intervals for each $30 \mathrm{~min}$ treatment period (Baseline, SPNO, Wash). The first point in each plot is time 0 . Cells were selected if they could be tracked throughout.

microglia, although for a distance $<10 \mu \mathrm{m}$, the cell nucleus alone rather than the entire cell might have moved (McGladeMcCulloh et al., 1989). Although the population of microglial cells was heterogeneous as to movement, the independent migration of each cell was inhibited during SPNO exposure. No cells (of 131 total in two cords) began moving during SPNO treatment.

Speed for each cell was calculated from the distance traveled in each 30 min treatment period, and an average velocity was calculated for each treatment group (Fig. 7). For a baseline, mean \pm SEM speed was measured to be $0.93 \pm 0.13 \mu \mathrm{m} / \mathrm{min}(n=9$ cells $)$. 


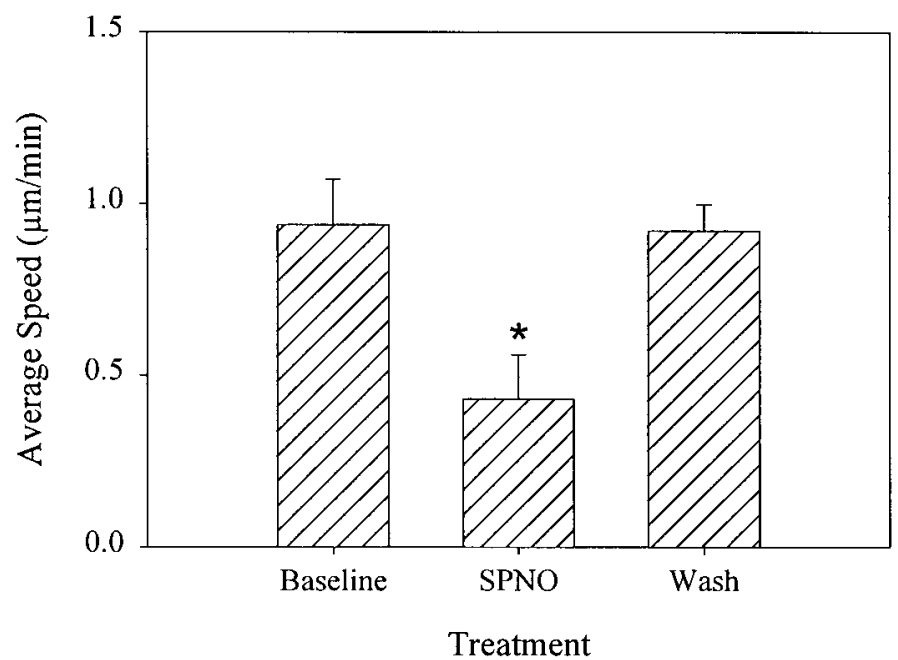

Figure 7. SPNO slows the migration of cells to a crush. Low-light fluorescence video microscopy was used to track individual Hoechststained microglia as they migrated to a crush, as in Figure $6(n=9$ cells from 2 cords). Selected cells remained in the same focal plane during each 30 min treatment period: baseline (Baseline), $1 \mathrm{~mm}$ SPNO (SPNO), and SPNO washout (Wash). A significant decrease in velocity was observed after SPNO exposure $(p<0.05)$. Cells recovered to baseline velocity after washout. Error bars are SEM. Comparisons were made using Kruskal-Wallis rank sum ANOVA $(p=0.008)$ and Dunnett's post hoc analysis at the $p=0.05$ level.

For all nine cells, including the six in Figure 6, movement slowed significantly to $0.43 \pm 0.12 \mu \mathrm{m} / \mathrm{min}$ during SPNO treatment $(p<$ $0.05)$, with a recovery to the baseline $(0.92 \pm 0.07 \mu \mathrm{m} / \mathrm{min})$ after washout of SPNO. Thus, SPNO blocked microglial cell accumulation by stopping or slowing cells migrating to the crush.

In contrast to this, slowing of migration caused by SPNO, $1 \mathrm{~mm}$ L-NAME, and $1 \mathrm{mM}$ D-NAME did not affect the migration rate of microglia. The average velocity of individual microglial cells in injured nerve cords treated with $1 \mathrm{mM}$ L-NAME was $10.2 \pm 2.0$ $\mu \mathrm{m} / \mathrm{min}$, whereas the average velocity in tissue treated with $1 \mathrm{mM}$ D-NAME was $8.8 \pm 1.4 \mu \mathrm{m} / \mathrm{min}$. Statistical analysis indicated that treatment with $1 \mathrm{mM}$ L-NAME compared with treatment with L-15 or $1 \mathrm{mM}$ D-NAME did not significantly affect the average velocity of migrating microglia (ANOVA; $F_{(2,18)}=0.434$; $p=0.6544 ; n=5)$.

To understand better the mechanisms by which microglia stopped moving in the presence of NO, movement of microglial filopodia and lamellipodia was observed directly in 3-D collagen gels. In gels, the entire cell can be viewed continuously and not only the nucleus in time lapse. Microglia migrated from explants of nerve cord into the gels and were viewed with phase optics for up to $10 \mathrm{~d}$ in culture. SPNO, added to a final concentration of 1 $\mathrm{mM}$ in L-15 culture medium layered on the gel, completely arrested movement of 10 of 21 cells examined and markedly slowed the movement of nine others, with no effect on two. For 15 cells that had slowed or stopped, washout reversed the effect in nine within the viewing time. An example is shown in Figure 8 in which movement was estimated by subtracting successive frames (top row, difference is white area in last frame). Movement of the lamellipodia stopped with the addition of $1 \mathrm{~mm}$ SPNO (Fig. 8, middle row) and resumed within minutes of washout (Fig. 8, bottom row). Addition of aged SPNO solutions as controls had no effect in three preparations examined (data not shown). In two preparations, the NO scavenger reduced-hemoglobin was added either $15 \mathrm{~min}$ before addition of SPNO or after incubation in SPNO for $80 \mathrm{~min}$, and in each case, the SPNO was without effect, indicating that NO specifically arrested cell movement.

\section{DISCUSSION}

NOS activity has been associated previously with the appearance of microglia at CNS lesions in the leech. The present paper demonstrates that microglia accumulate after early expression of NOS activity and eNOS immunoreactivity. Microglia distributed throughout the CNS at the time of injury are one source of NOS activity (Shafer et al., 1998). The timing and location of NO production, immediately at the site of injury and before microglial accumulation, are consistent with a role for NO in modulating migration of the cells. In this study of the expression of eNOS immunoreactivity, the density of cells within the crush peaks by $24 \mathrm{hr}$ after injury. Later, cells also accumulate adjacent to the lesion (Morgese et al., 1983). The high density persists up to 8 weeks after injury, although synapses have already reformed. The injury that severs axons also damages the connective glial cell; after months, the glia degenerate and microglia ensheathe the axons (Elliott and Muller, 1981).

SPNO acts directly to reduce accumulation of microglia at the lesion by blocking or reducing migration, not through a cytotoxic effect. The inhibitory effect of SPNO was blocked by the NO scavenger carboxy-PTIO, and partially expired SPNO was less effective than fresh SPNO at blocking migration. Aged SPNO might possess toxic NO breakdown products such as peroxynitrites, but these would be expected to increase with time and, if the inhibition were primarily caused by these by-products, then aged SPNO should have enhanced block of accumulation, which it did not. Instead, the blocking effect was reduced, consistent with reduced levels of NO. Third, the effect of SPNO could be reversed by washing either before or after crushing. Both inactive and activated microglia responded to SPNO in a manner inconsistent with cytotoxicity. L-NAME too acted specifically to reduce accumulation, although not by stopping migrating microglia, as the inactive enantiomer D-NAME did not affect accumulation.

Previous work with low-light video microscopy has shown that microglia travel individually to lesions, not moving uniformly as a herd, and that at any time no more than $20 \%$ of microglia near a lesion are moving (McGlade-McCulloh et al., 1989). As a population, the microglia also appear to be heterogeneous in their response to exogenous NO. Some cells stopped completely and others showed varying amounts of slowing. This is consistent with variability in the arrest of movement by cultured microglia upon addition of SPNO. Although the source of these differences is unknown, in mammals, different types of injury can elicit different activation states (Kreutzberg, 1996). Moreover, a single injury can yield differential gene expression in mammalian microglial cell populations (Elkabes et al., 1996; Reichert and Rotshenker, 1996; Tanaka et al., 1998). A variety of other signals might also be expected to act on migrating microglia and be unevenly distributed in the nervous system (Smith et al., 1987). Despite differences among cells, however, a general inhibitory action of NO was observed for the entire group.

It is possible that the injury-induced NO acts as a stop signal for migrating microglia. According to this hypothesis, the highest concentrations of NO would be at the crush, which would stop the migration of cells, presumably to repair damaged tissue. Both our accumulation and low-light video microscopy studies support this notion. The NO donor SPNO increased NO levels all along the cord, which reversibly arrested migration, even outside the crush. 
0
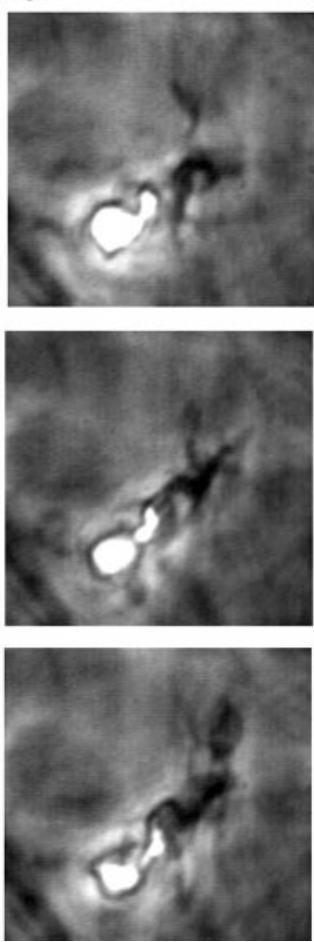

5
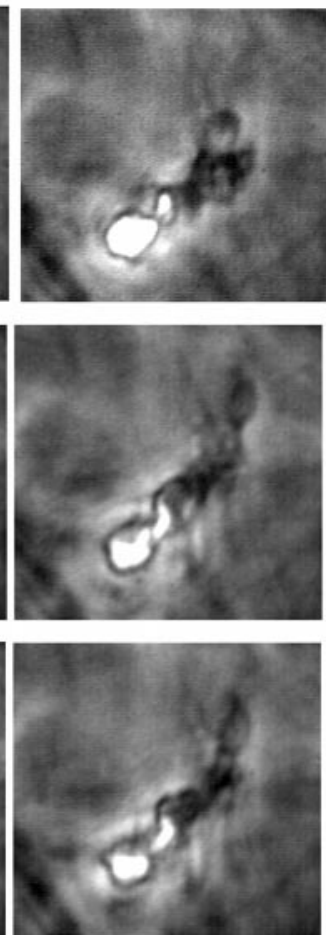

10
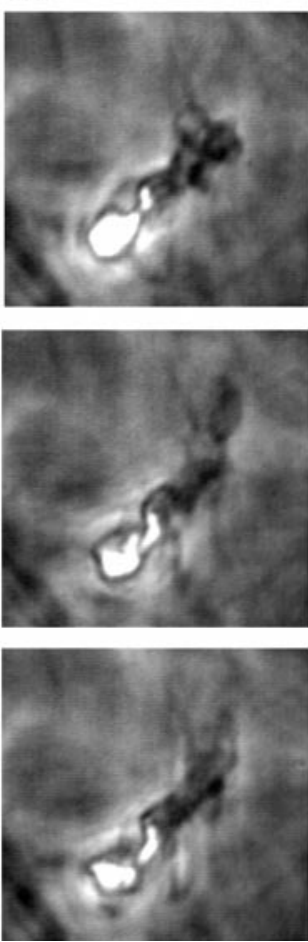

15 minutes
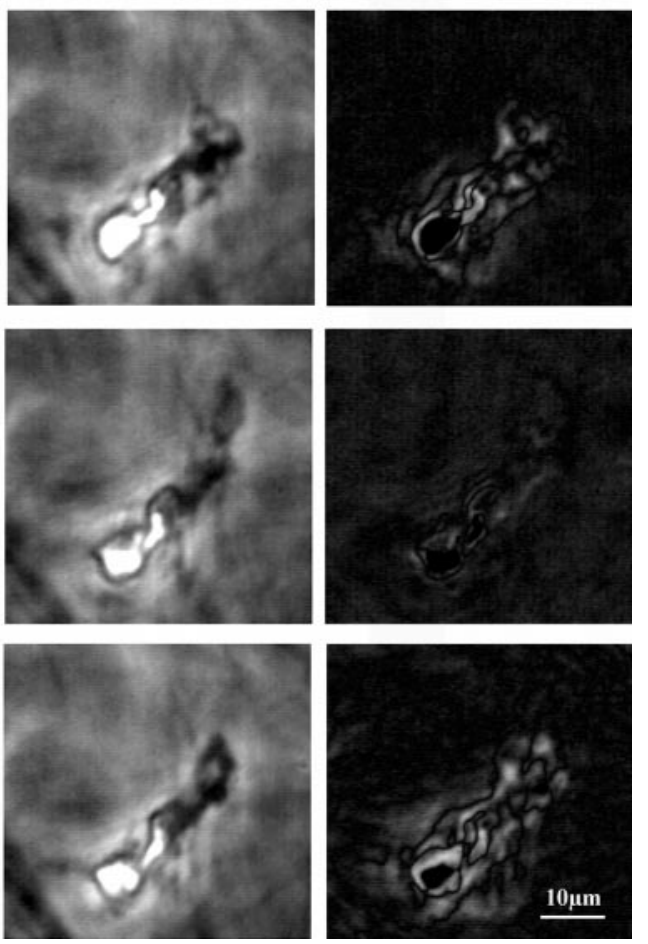

Figure 8. NO donor SPNO reversibly arrested movement of microglial cells in three-dimensional collagen gels. Microglia that migrated into the gels moved their lamellipodia with little translocation. Phase contrast images in each row were separated by 5 min intervals; right panels are computergenerated subtractions of the last two images and show cell movement in $5 \mathrm{~min}$ in white. Addition of SPNO to a final concentration of 1 mM in L-15 culture medium arrested movement of lamellipodia (middle row), whereas addition of aged NO donor solutions (data not shown) was without effect. The usual motility resumed within minutes of washout (bottom row).

It appears that application of SPNO to the entire cord stopped the cells in place, effectively preventing them from migrating to the crush, resulting in reduced microglial accumulation at the lesion site. Incubation of crushed cords in the NOS inhibitor L-NAME reduced NO levels and microglial cell accumulation but did not affect the migration rate or population of microglia moving. Low-light video recordings in the presence of L-NAME suggest that, in the absence of $\mathrm{NO}$, microglia continue to migrate and do not accumulate at the lesion, supporting the hypothesis that injury-induced NO serves as a stop signal for migrating microglia.

Several substances, including NO, have been shown to inhibit movement or growth at one concentration and have an opposite, promoting effect at other concentrations. In migrating neutrophils, low concentrations of NO can enhance movement and higher concentrations inhibit movement (VanUffelen et al., 1996, 1998). Similarly opposing effects of NO have been observed in neuronal growth cones of Helisoma (Van Wagenen and Rehder, 1999) and growing neurites in rat dorsal root ganglion (Tsukada and Fukuda, 1998). Also in growth cones, intracellular $\left[\mathrm{Ca}^{2+}\right]$ (Kater and Mills, 1991; Rehder and Kater, 1992) and [cAMP] (Song et al., 1997) have each been proposed to provide such a dual regulation of extension. We have not completely ruled out a dual role for NO in leech microglial migration in which high concentrations of NO at the crush would stop migration, whereas lower concentrations at a distance in which microglial cells are migrating might promote it. NO application can halt microglial migration, but a chemoattractive role for NO is less clear because blocking NO production does not interfere with injury-induced movement of microglial cells. Other attractive agents, however, might act in addition to $\mathrm{NO}$ and continue to drive cells to the crush.

The effects of NO on nerve repair and regeneration extend beyond influencing microglia, because NO has been shown to influence the movement of growing axons. In addition to the examples mentioned above, NO halted neurite motility in chick dorsal root ganglion explants (Hess et al., 1993) and collapsed growth cones in retinal ganglion axons (Rentería and Constantine-Paton, 1995). Conversely, in the presence of NOS inhibitors, retinal axons grew past their targets (Wu et al., 1994; Cramer et al., 1996; Gibbs and Truman, 1998). Thus, NO might hold axons close to their point of synapse until permanent connections form, might be a retrograde signal for activity-dependent pruning of synapses (Williams et al., 1994; Wu et al., 1994; Wang et al., 1995), or both. Because microglia themselves produce NO at both early and late stages of repair, they are likely to sculpt repair by both promoting and inhibiting axon growth.

\section{REFERENCES}

Angelov DN, Walther M, Streppel M, Guntinas-Lichius O, Neiss WF, Probstmeier R, Pesheva P (1998) Tenascin-R is antiadhesive for activated microglia that induce downregulation of the protein after peripheral nerve injury: a new role in neuronal protection. J Neurosci 18:6218-6229.

Barron KD (1995) The microglial cell. A historical review. J Neurol Sci 134:57-68.

Blackshaw SE, Arkison S, Cameron C, Davies JA (1997) Promotion of regeneration and axon growth following injury in an invertebrate nervous system by the use of three-dimensional collagen gels. Proc R Soc Lond B Biol Sci [Erratum (1997) 264:1101-2] 264:657-661. 
Blottner D, Grozdanovic Z, Gossrau R (1995) Histochemistry of nitric oxide synthase in the nervous system. Histochem J 27:785-811.

Camhi JM, Macagno E (1991) Using fluorescence photoablation to study the regeneration of singly cut leech axons. J Neurobiol 22:116-129.

Chen A, Sahley C, Muller KJ (1998) An effect of nitric oxide on microglial cell accumulation in the injured central nervous system of the leech. Soc Neurosci Abstr 24:67.

Cramer KS, Angelucci A, Hahm JO, Bogdanov MB, Sur M (1996) A role for nitric oxide in the development of the ferret retinogeniculate projection. J Neurosci 16:7995-8004.

Elkabes S, DiCicco-Bloom EM, Black IB (1996) Brain microglia/macrophages express neurotrophins that selectively regulate microglial proliferation and function. J Neurosci 16:2508-2521.

Elliott EJ, Muller KJ (1981) Long-term survival of glial segments during nerve regeneration in the leech. Brain Res 218:99-113.

Frenkel SR, Clancy RM, Ricci JL, DiCesare PE, Rediske JJ, Abramson SB (1996) Effects of nitric oxide on chondrocyte migration, adhesion, and cytoskeletal assembly. Arthritis Rheum 39:1905-1912.

Gibbs SM, Truman JW (1998) Nitric oxide and cyclic GMP regulate retinal patterning in the optic lobe of Drosophila. J Neurosci 20:83-93.

Guthrie S, Lumsden A (1994) Collagen gel coculture of neural tissue. Neuroprotocols 4:116-120.

Hess DT, Patterson SI, Smith DS, Skene JH (1993) Neuronal growth cone collapse and inhibition of protein fatty acylation by nitric oxide. Nature 366:562-565.

Kater SB, Mills LR (1991) Regulation of growth cone behavior by calcium. J Neurosci 11:891-899.

Kreutzberg GW (1996) Microglia: a sensor for pathological events in the CNS. Trends Neurosci 19:312-318.

Magazine HI, Liu Y, Bilfinger TV, Fricchione GL, Stefano GB (1996) Morphine-induced conformational changes in human monocytes, granulocytes, and endothelial cells and in invertebrate immunocytes and microglia are mediated by nitric oxide. J Immunol 156:4845-4850.

McGlade-McCulloh E, Morrissey AM, Norona F, Muller KJ (1989) Individual microglia move rapidly and directly to nerve lesions in the leech central nervous system. Proc Natl Acad Sci USA 86:1093-1097.

Morgese VJ, Elliott EJ, Muller KJ (1983) Microglial movement to sites of nerve lesion in the leech CNS. Brain Res 272:166-170.

Nicholls JG (1987) The search for connections: study of regeneration in the nervous system of the leech. Magnes lecture series, Vol II, pp 1-84. Sunderland, MA: Sinauer.

Nicholls JG, Baylor DA (1968) Specific modalities and receptive fields of sensory neurons in the CNS of the leech. J Neurophysiol 31:740-756.

Ready DF, Nicholls J (1979) Identified neurones isolated from leech CNS make selective connections in culture. Nature 281:67-69.

Rehder V, Kater SB (1992) Regulation of neuronal growth cone filopodia by intracellular calcium. J Neurosci 12:3175-3186.

Reichert F, Rotshenker S (1996) Deficient activation of microglia during optic nerve degeneration. J Neuroimmunol 70:153-161.

Rentería RC, Constantine-Paton M (1995) Exogenous nitric oxide causes collapse of retinal ganglion cell axonal growth cones in vitro. J Neurobiol 29:415-428.

Sarkar R, Meinberg EG, Stanley JC, Gordon D, Webb RC (1996) Nitric oxide reversibly inhibits the migration of cultured vascular smooth muscle cells. Circ Res 78:225-230.

Shafer OT, Chen A, Kumar SM, Muller KJ, Sahley CL (1998) Injuryinduced expression of endothelial nitric oxide synthase by glial and microglial cells in the leech central nervous system within minutes after injury. Proc R Soc Lond B Biol Sci 265:2171-2175.

Smith PJS, Howes EA, Treherne JE (1987) Mechanisms of glial regeneration in an insect central nervous system. J Exp Biol 132:59-78.

Song HJ, Ming GL, Poo MM (1997) cAMP-induced switching in turning direction of nerve growth cones. Nature 388:275-279.

Stewart RR (1994) Membrane properties of microglial cells isolated from the leech central nervous system. Proc R Soc Lond B Biol Sci 255:201-208.

Tanaka S, Suzuki K, Watanabe M, Matsuda A, Tone S, Koike T (1998) Upregulation of a new microglial gene, $m r f-1$, in response to programmed neuronal cell death and degeneration. J Neurosci 18:6358-6369.

Thanos S, Mey J, Wild M (1993) Treatment of the adult retina with microglia-suppressing factors retards axotomy-induced neuronal degradation and enhances axonal regeneration in vivo and in vitro. J Neurosci 13:455-466.

Tsukada S, Fukuda J (1998) Nitric oxide induced both promotion and inhibition for neurite growth in rat DRG neurons. Soc Neurosci Abstr 24:1283.

Van Wagenen S, Rehder V (1999) Regulation of neuronal growth cone filopodia by nitric oxide. J Neurobiol 39:168-185.

VanUffelen BE, de Koster BM, Van den Broek PJA, VanSteveninck J, Elferink JGR (1996) Modulation of neutrophil migration by exogenous gaseous nitric oxide. J Leukocyte Biol 60:94-100.

VanUffelen BE, Van der Zee J, de Koster BM, VanSteveninck J, Elferink JGR (1998) Intracellular but not extracellular conversion of nitroxyl anion into nitric oxide leads to stimulation of human neutrophil migration. Biochem J 330:719-722.

von Bernhardi R, Muller KJ (1995) Repair of the central nervous system: lessons from lesions in leeches. J Neurobiol 27:353-366.

Wang T, Xie Z, Lu B (1995) Nitric oxide mediates activity-dependent synaptic suppression at developing neuromuscular synapses. Nature 374:262-266.

Williams CV, Nordquist D, McLoon SC (1994) Correlation of nitric oxide synthase expression with changing patterns of axonal projections in the developing visual system. J Neurosci 14:1746-1755.

Wu HH, Williams CV, McLoon SC (1994) Involvement of nitric oxide in the elimination of a transient retinotectal projection in development. Science 265:1593-1596.

Yao J, Schoecklmann HO, Pröls F, Gauer S, Sterzel RB (1998) Exogenous nitric oxide inhibits mesangial cell adhesion to extracellular matrix components. Kidney Intl 53:598-608. 